\title{
A source of illumination for low-noise 'Violin-Mode' shadow sensors, intended for use in interferometric gravitational wave detectors
}

\author{
N.A. Lockerbie, K.V. Tokmakov, and K. A. Strain ${ }^{1}$ \\ SUPA (Scottish Universities Physics Alliance), Department of Physics, \\ University of Strathclyde, 107 Rottenrow, Glasgow G4 0NG, UK
}

\begin{abstract}
A low-noise source of illumination is described for shadow-sensors having a displacement sensitivity of $(69 \pm 13)$ picometres $(\mathrm{rms}) / \sqrt{ } \mathrm{Hz}$, at $500 \mathrm{~Hz}$, over a measuring span of $\pm 0.1 \mathrm{~mm}$. These sensors were designed to detect 'Violin-Mode' resonances in the suspension fibres of the test-masses/mirrors for the Advanced LIGO (Laser Interferometer Gravitational wave Observatory) gravitational wave detectors. The source of illumination (emitter) described here used a single column of $8 \times$ miniature Near InfraRed LEDs $(\lambda=890 \mathrm{~nm})$. These emitters cast the shadows of $400 \mu \mathrm{m}$ diameter fused silica suspension fibres onto their complementary shadowdisplacement detectors, located at a distance of 74 fibre diameters $(29.6 \mathrm{~mm})$ behind the axes of the fibres themselves. Violin-Mode vibrations of each fibre were sensed as differential $A C$ photocurrents in the corresponding 'split-photodiode' detector. This paper describes the design, construction, noise analysis, and measures that were taken in the conception of the emitters, in order to produce high-contrast shadows at such distant detectors. In this way it proved possible to obtain, simultaneously, a very high transfer sensitivity to Violin-Mode vibration of the fibres, and a very low level of detection noise-close to the fundamental shot noise limit—whilst remaining within the constraints of this simple design of emitter. The shadow detector is described in an accompanying paper.
\end{abstract}

PACS numbers: 04.80.Nn, 84.30.-r, 06.30.Bp, 07.07.Df, 07.57.-c

\section{Introduction}

Each $40 \mathrm{~kg}$ test-mass/mirror, in the Advanced LIGO 'aLIGO' ground-based interferometric gravitational wave detectors, is to be hung in vacuum from a multi-stage pendulum seismic isolation suspension [1-4]. The lowest pendulum stage of each such suspension will comprise four fused-silica suspension fibres, each measuring approximately $600 \mathrm{~mm}$ long, by $400 \mu \mathrm{m}$ in diameter, with the fibres' lower ends being attached to the test-mass/mirror, itself $[4,5]$.

Earthquakes and other seismic disturbances, sudden relaxations of mechanical stress, etc., can excite the lateral Eigenmodes, or 'Violin-Modes,' of these silica suspension fibres. Given the fundamental modal frequencies of order $500 \mathrm{~Hz}$, and the very high Q-values $\left(\sim 6 \times 10^{8}[6]\right)$, these resonances, and their harmonics, may transfer vibrational energy to their suspended test masses at frequencies lying within the gravitational wave detection bandwidth. Therefore, it is necessary to monitor fibre motions [7] and then to cold-damp Violin-Mode resonances [8].

The Violin-Mode (VM) monitoring system, whose LED-based low-noise sources of illumination are described here, was conceived as a four-channel shadow-sensor, having a dedicated displacement sensor for each suspension fibre. In this way, it would be possible to monitor simultaneously the VM signals from all four fibres of a fully-suspended test-mass. The target VM displacement sensitivity was $100 \mathrm{pm}(\mathrm{rms})$ per $\sqrt{\mathrm{Hz}}$ at $500 \mathrm{~Hz}$, together with (ultimately) a detection span, or sensing range, of $\pm 0.1 \mathrm{~mm}$ about the mean position of each fibre-in order to compensate for drift over time in the mean position of the fibre, due to slow mechanical relaxation. In this work each shadow-sensor comprised an 'emitter,' in the form of a LED-based source of Near InfraRed (NIR) illumination for its respective fibre $(\lambda=890 \mathrm{~nm})$, and a 'split-photodiode' based (two-element) shadow-displacement-sensing detector. In

\footnotetext{
${ }^{1}$ University of Glasgow, Institute for Gravitational Research, SUPA, School of Physics and Astronomy, Kelvin Building, University Avenue, Glasgow, G12 8QQ, Scotland, UK.
} 
practice, attaining the required level of $V M$ displacement sensitivity proved challenging, and much effort went into the design of both the emitters and detectors of this work, in order to maximize the raw displacement sensitivity at $500 \mathrm{~Hz}$ (plus $\sim 10$ harmonics) of this simple shadow sensor, and, at the same time, to 'beat-down' all potential sources of interfering noise. The VM bandwidth of the sensor, determined by its transimpedance amplifier, was $226 \mathrm{~Hz}-$ $8.93 \mathrm{kHz}$, at the $-3 \mathrm{~dB}$ points.

The emitters turned out to have a profound influence on the final, attainable, VM displacement sensitivity. Careful attention therefore was paid to their design-described below-and a VM displacement sensitivity of $(69 \pm 13)$ picometres $(\mathrm{rms}) / \sqrt{ } \mathrm{Hz}$, at $500 \mathrm{~Hz}$, was achieved, even at the end-of span positions. The detectors are described in an accompanying paper [9].

\section{Overview of the Violin-Mode shadow sensor system}

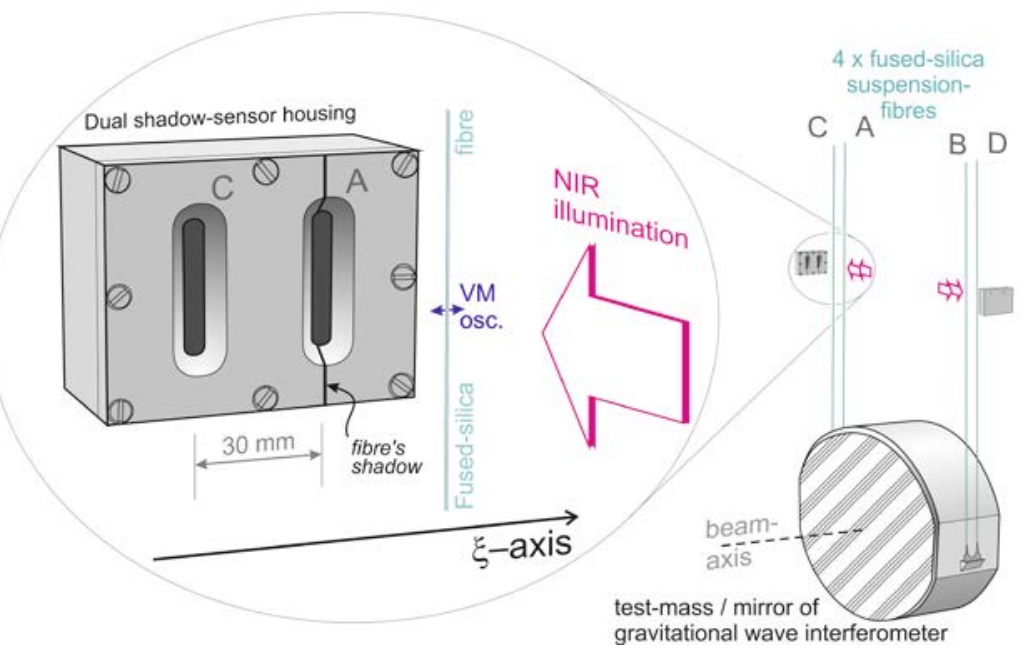

Fig.1. The aLIGO suspension system for a $40 \mathrm{~kg}$ test-mass/mirror comprises four fused-silica suspension fibres, arranged in two pairs across the $340 \mathrm{~mm}$ diameter of the test-mass. Here, the paired fibres have been labelled $(C, A)$ and $(B, D)$. For clarity, the enlargement in the schematic shows only fibre ' $A$ ' of the $(C, A)$ pair, together with the collimated source of NIR illumination $(\lambda=890 \mathrm{~nm})$ from its particular emitter. The resulting shadow cast by this fibre falls over the right-hand aperture in the front-plate of the Dual shadow-sensor housing ' $C$ - $A$ ' of this work, as indicated-as well as over an internally-mounted 'split-photodiode' detector. Here, VM oscillations of the fibre, indicated by the double-headed arrow, are transferred to its shadow without any lateral magnification. Similarly, the shadow of fibre $\mathrm{C}$ would fall over the left-hand aperture in the housing's front-plate, and over its respective internally-mounted sensor. The cylindrical aLIGO suspension fibres measure $600 \mathrm{~mm}$ long by $400 \mu \mathrm{m}$ in diameter.

In this work the four suspension fibres of a test-mass, together with their respective emitters and shadow-sensors (detectors), have been labelled A-D, as indicated in Figure 1. Each pair of suspension fibres ( $C$ and $A)$, and ( $D$ and $B$ ), were themselves physically separated by a nominal $30 \mathrm{~mm}$ parallel to the $\xi$-axis (i.e., parallel to the beam-axis of the gravitational wave interferometer, and the cylindrical axis of the test-mass/mirror).

\section{Low-noise emitters (sources of illumination for the silica suspension fibres)}

There is negligible optical absorption in a fused silica fibre measuring $400 \mu \mathrm{m}$ in diameter, over a wavelength range for incident light of $200 \mathrm{~nm}-2 \mu \mathrm{m}$. The shadow cast by such a fibre, when illuminated from its side, depends instead primarily on strong refraction, and a very small amount of reflection, at the fibre's surface. Consequently, a wide range of illuminating wavelengths might have proved equally suitable for the emitters of this work-with the exception of the $1064 \mathrm{~nm}$ (and $532 \mathrm{~nm}$ ) to be used by the aLIGO interferometers, of course.

It was decided to avoid high-frequency modulation of these emitters as a potential route to obtaining greater displacement sensitivity for the detection of $V M$ fibre oscillations. This 
choice was made in order to avoid the possibility of interference, however remote, with the optical sensing of the gravitational wave interferometers. Instead, steady, unmodulated, lowintensity illuminating beams in the Near InfraRed were chosen, where such wavelengths would be well-matched to silicon photodiode-based shadow-sensors. Early work, employing NIR Laser-diodes as the sources of illumination, highlighted the need to avoid fringing effects at the borders of the fibres' shadows, which otherwise could compromised the shadow-displacement sensitivity of the (necessarily) narrow split-photodiode detectors used. It was decided, therefore, that incoherent sources in the form of NIR LED-based emitters should be used to illuminate the suspension fibres.

The shadow-displacement detectors of this work were constrained to being located at some stand-off distance from the fibres whose $V M$ vibrations were to be monitored-at an optical path-length (via a right-angled reflection) of 74 fibre diameters $(29.6 \mathrm{~mm})$ from the fibres' axes, as it transpired, albeit at a physical stand-off distance of $12.5 \mathrm{~mm}$ from each detector housing's front-plate. This was necessary in order to prevent accidental touches to the fibres during retro-fitting of the $V M$ monitoring system, and the ensuing, certain, shattering of the highly-tensioned suspension fibres. Early work carried out on the displacement sensors for OSEMs (Optical Sensing ElectroMagnets in aLIGO) showed that the emitted beams from lensed NIR LEDs (OP232s [11]) wandered somewhat in direction-certainly at frequencies up to $10 \mathrm{~Hz}$ [12]. Any beam-wandering from such an illuminating LED would have added transverse displacement noise to the position of the fibre's distant shadow, thereby mimicking lateral movement of the illuminated fibre, itself. Additionally, it was discovered that large diameter LED sources, such as the OP232 (lens dia. $=3.9 \mathrm{~mm}$ ), would cast their illumination around a relatively thin target fibre (dia. $=0.4 \mathrm{~mm}$ ), so as to create a shadow in the plane of the detector consisting of a rather broad, diffuse, penumbra, with poor contrast. Also, the available space for the full VM detection system was physically quite constrained, and placing such a LED source much further away from its fibre, in order to mitigate the shadow delineation problem, was not an option.

Shadow contrast, or 'depth of shadow,' as well as intensity of illumination, turned out to be important parameters in connection with maximising shadow displacement sensitivity, as explained in the accompanying paper [9]. Therefore, for the reasons given above, it was decided not to use single large, powerful, LEDs in each emitter source, but, instead, to use: -

i) sources in the form of a linear column of $\mathrm{N}$ individual LEDs, in order to reduce any shadow displacement noise due to beam-wandering - potentially, by a factor of $\sqrt{ } \mathrm{N}$,

ii) lensed LEDs of intrinsically small physical diameter (OP224s [11]), having a lens diameter $=1 \mathrm{~mm}$ ), and

iii) LEDs located sufficiently far from the illuminated fibre that they subtended, effectively, a small angular width at the fibre $\left(\sim 1^{\circ}\right)$ — similar to the angular width of the fibre subtended at the detector, in fact.

The consequential reduction in irradiance at the split-photodiode detectors due to iii) was compensated, to some extent, by the increase in radiant flux from the multiple sources of i); and the optimal (minimum) number of LEDs in each emitter was determined by experiment to be $\mathrm{N}=8$, in order to meet the target $V M$ detection sensitivity of $100 \mathrm{pm}(\mathrm{rms}) / \sqrt{\mathrm{Hz}}$, at $500 \mathrm{~Hz}$ - the $V M$ displacement sensitivity increasing as $\sqrt{ } \mathrm{I}_{0}$ i.e., as $\sqrt{ } \mathrm{N}$, where $\mathrm{I}_{0}=$ irradiance at the detector [9].

The shadow-sensor's emitter described here was adapted from an earlier version, which was intended to have a vacuum-tight housing, and which had a step-wise steerable output beam ${ }^{2}$.

\footnotetext{
${ }^{2}$ In the original specification for the $V M$ detection system the functional span (measuring range) was to be $\pm 0.5 \mathrm{~mm}$ about the mean position of each fibre. Nevertheless, the $V M$ detection sensitivity over this extended range was to be $100 \mathrm{pm}(\mathrm{rms}) / \sqrt{ } \mathrm{Hz}$, at $500 \mathrm{~Hz}$, and, in order to cover this $140 \mathrm{~dB}$ dynamic range, a source comprising five closely adjacent columns of 8 LEDs was conceived, for each emitter. Ultimately the detection
} 
MST-101172.R2

Consequently, the housing was made thick-walled, in order to withstand the differential pressure. This '5-LED-column' emitter could be used to produce a fan of 5 collimated illuminating NIR beams, with adjacent emitted beams being angled at just $\pm 0.63^{\circ}$ relative to each other, via a 'reverse Galilean' lens system [10]. In use, the columns of LEDs could be switching on, one at a time, in order to displace slightly the illuminated fibre's shadow-in a step-wise fashion. In this way the fibre's shadow could be re-positioned back onto its fixed detector, had the fibre drifted in position over time.
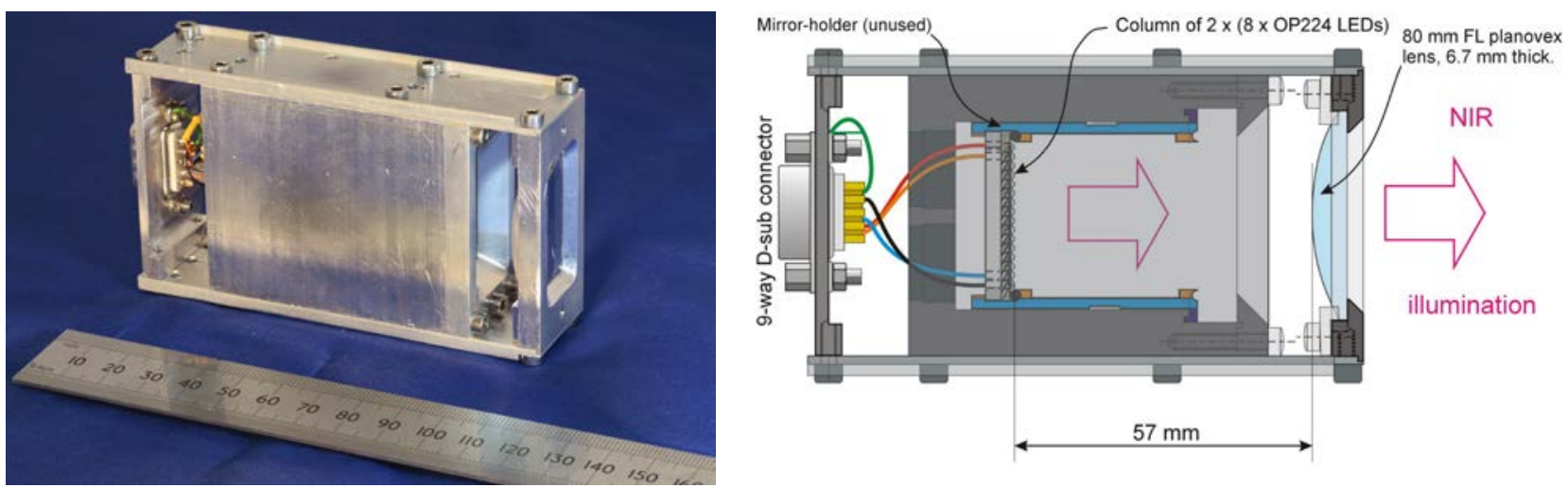

Figure 2. Left: photo of a single LED-based illumination source (emitter), derived from an original '5-LEDcolumn' version (please refer to the text). Right: functional schematic of the internal parts of the emitter, showing the single column of $2 \times(8 \times$ OP224) LEDs, and the $80 \mathrm{~mm}$ Focal Length (FL) spherical plano-convex collimating lens-which was cropped so as to fit into its $25 \mathrm{~mm} \times 40 \mathrm{~mm}$ lens holder. The block arrows indicate the direction of the emitted NIR beam $(\lambda=890 \mathrm{~nm})$ coming from the vertical, essentially linear, source of illumination. The column of LEDs was in fact arranged as two separate series-connected strings of 8 LEDs, alternate LEDs belonging to the same string (for redundancy: one string of LEDs was held in reserve, against possible failure of the primary string). The illuminated fused silica fibres were located at a distance of $15 \mathrm{~mm}$ from the front faces of their respective emitter housings. Four such emitters were built, one for each silica fibre of a full suspension.

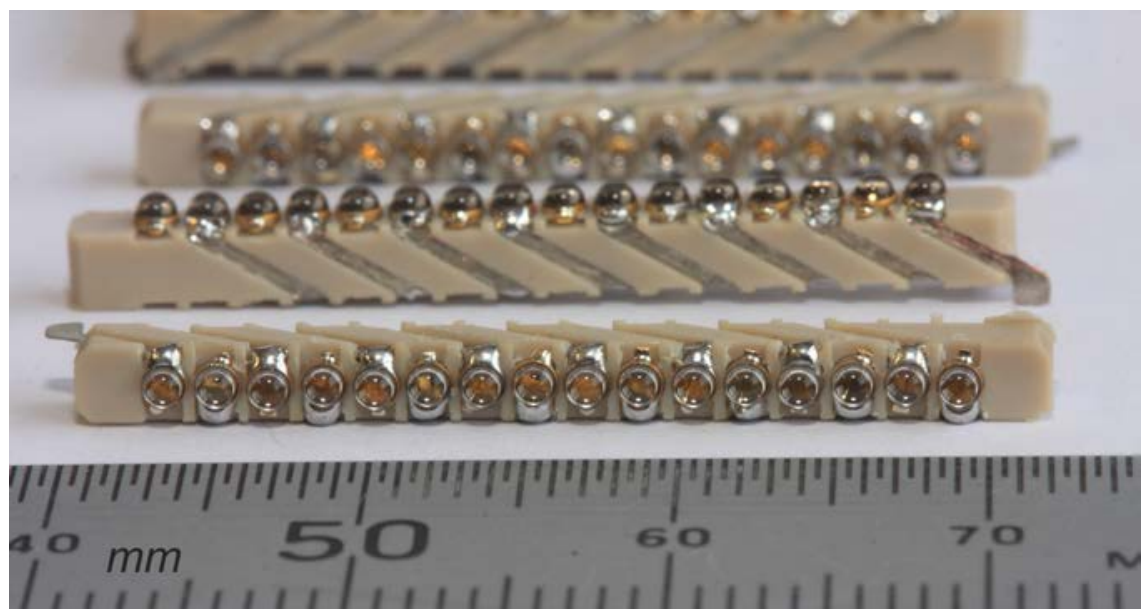

Figure 3. The photo shows the bars (columns) of $2 \times(8 \times$ OP224) NIR LEDs which were used in this work as the source of illumination for the fused silica suspension fibres, and their VM shadow detectors. Originally intended for 5-column sources, each column comprised two separate sets of $8 \times$ LEDs, alternate LEDs being connected in series using tin-plated copper strips, $0.1 \mathrm{~mm}$ thick. The LEDs and their connecting strips were set into holders machined from insulating PEEK material.

These original (thick-walled) emitter housings were adapted subsequently for the emitter having the single column of LEDs, and one of the resulting emitters is shown both as a photo, and as a sectional schematic, in Figure 2. A photo of the columns (or bars) of LEDs is shown

span was reduced to $\pm 0.1 \mathrm{~mm}$, and this allowed the single column of LEDs described here to be employed, which also could be moved much closer to the illuminated fibre-since the full telescopic lens system was no-longer needed. Front-silvered mirrors, mounted above and below the five columns of LEDs, also were removed from the emitter housing, as was a cylindrical plano-concave diverging lens, which was to serve also as an interior vacuumtight window to the housing. The single converging lens of the telescope was retained, however. 
in Figure 3, where the OP224 'pill' package NIR LEDs are seen to be equally-spaced along each bar with, ultimately, the LEDs' emission axes all being horizontal. The overall physical height of the LED columns was $26 \mathrm{~mm}$ (measured over the LEDs; and the PD sensors in each detector were closely matched, at $29.1 \mathrm{~mm}$ high). In the Figure the miniature OP224 LEDs' converging lenses can be seen, the lenses measuring approximately $1 \mathrm{~mm}$ in diameter. For the single column of LEDs that actually was used, the LEDs could have been set, more conveniently, into a double-sided (copper-plated) ceramic printed circuit board.

In terms of their spectral output the Optek OP224 LEDs have a published line width at their half-power points of $80 \mathrm{~nm}$ (50 mA forward- current) about their nominal $890 \mathrm{~nm}$ wavelength, and a temperature sensitivity of emitted wavelength equal to $0.18 \mathrm{~nm} /{ }^{\circ} \mathrm{C}$. Their matching Hamamatsu S2551 silicon photodiode detectors, on the other hand, have a photocurrent sensitivity to wavelength of approximately $0.06 \% / \mathrm{nm}$, at $890 \mathrm{~nm}$. Thus, taken together, their coupled sensitivity to these wavelength effects alone equates to a relatively negligible $0.01 \% /{ }^{\circ} \mathrm{C}$.

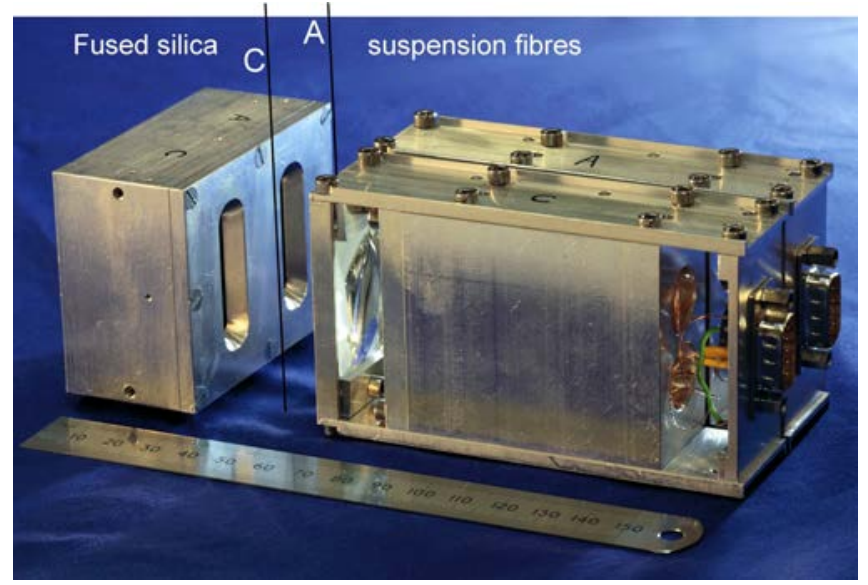

Figure 4. Photo showing the actual VM detection system, illustrated schematically in Figure 1, for one side of the test-mass suspension: one Dual shadow-sensor (detector) housing, and two, separate, NIR LED sources of illumination (emitters) are seen. The emitters were used to cast the fibres' shadows onto their respective detectors, and the composite photo shows the approximate positions of two fused-silica suspension fibres, labelled C and A. Here, the face-to-face separation of the emitter and detector housings was $27.5 \mathrm{~mm}$; but this distance could have been increased to $40 \mathrm{~mm}$, without loss of sensitivity to Violin-Mode shadow (fibre) vibrations (please refer to the text).

No mass budget for the VM detection system had been given in the original specification, and, the all-up mass of each assembled emitter was $0.26 \mathrm{~kg}$, although in a new design it could be reduced straightforwardly to $0.075 \mathrm{~kg}$, or less, with no need for a thick-walled housing to withstand the differential pressure of 1 atmosphere. Advanced LIGO has very stringent vacuum compatibility requirements, and, naturally, any adopted emitter’s LEDs, LED holders, connectors, and internal wiring, would all have to be compatible with these.

In the composite photo of Figure 4 a pair of single-LED-column emitters is seen, together with a (dual- ) detector housing, this assembly representing the $V M$ detection system for one side of an aLIGO suspension. The approximate positions of two fused silica suspension fibres are also indicated in the Figure.

\section{Practical shadow alignment}

The two silica fibres on each side of an aLIGO test-mass/mirror are spaced a nominal $30 \mathrm{~mm}$ apart $( \pm 0.5 \mathrm{~mm})$, whereas the corresponding detectors of this work were fixed, within a single detector Housing, at a separation of $30.0 \mathrm{~mm}$. The method of compensating for any variations in the nominal, horizontal, pitch of the suspension fibres required a simple adjustment to be made to the relative azimuthal orientations of the two individual emitter housings, on each side 
MST-101172.R2

of the suspension. The order of adjustment is indicated in Figure 5. Please note that during alignment any small-amplitude, low-frequency, 'pendulum-mode' motion of a suspended testmass, and its suspension fibres, was positively beneficial, since it assisted in the accurate centring of each shadow onto its respective detector [13]. This centring was achieved for each emitter/Fibre/detector by maximizing the amplitudes of the $180^{\circ}$ out-of-phase 'anti-phase' $\sim 0.6 \mathrm{~Hz}$ pendulum-mode signals seen at the detector Amplifier's VDC, $\mathrm{a}$ and $\mathrm{VDC}, \mathrm{b}$, outputs,

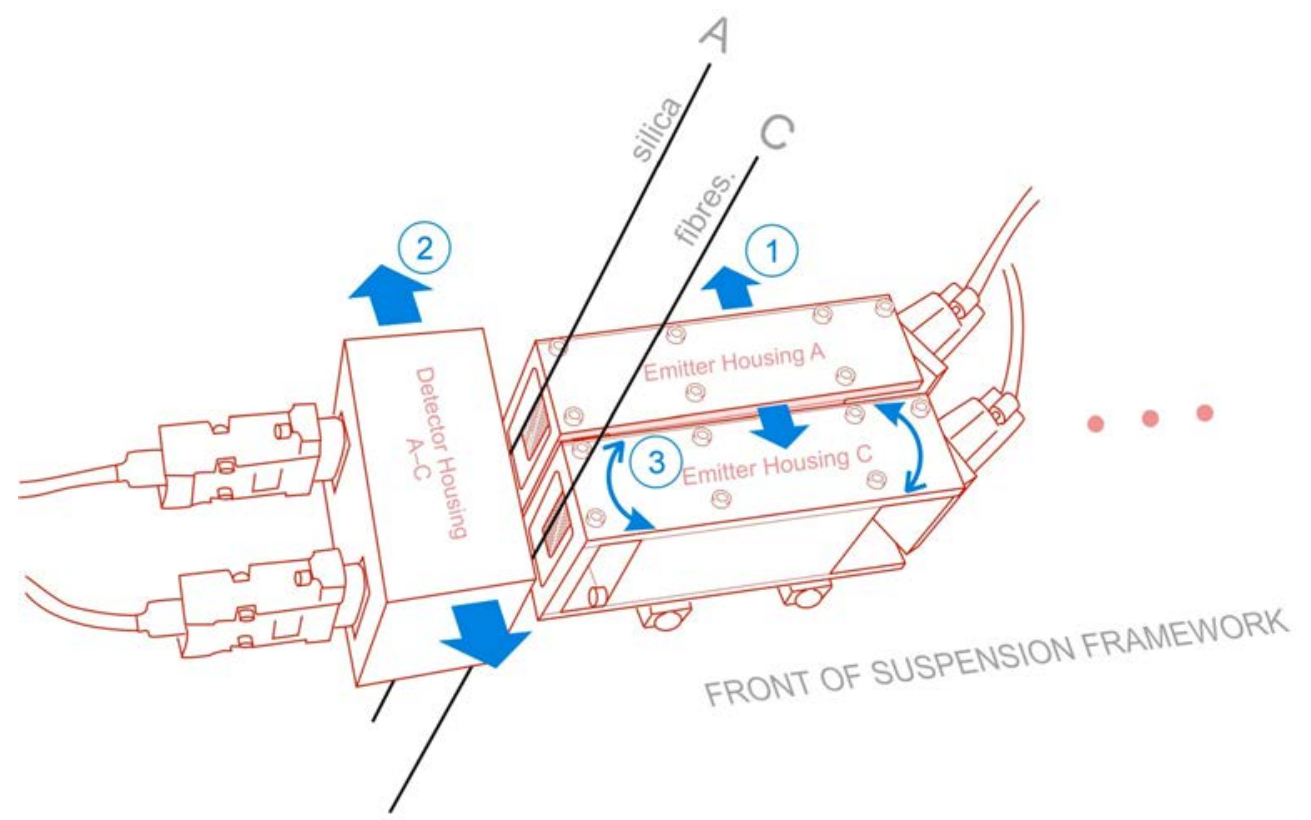

Figure 5. Schematic showing the practical 3-step procedure for aligning the Violin Mode emitters with their respective fused-silica fibres (shown in black) - here for two suspension fibres labeled A and C-and their respective detectors. In the Figure, the rear (male) D-sub connector panels of the emitter housings are shown angled, when compared with Figure 4, so as to allow their connecting cables to pass more easily to their current sources, shown schematically in Figure 6, via the rear of an actual suspension framework. Electrical connection to detectors $A$ and $C$ was via separate (female) 9-way D-sub connectors, as shown in the Figure. Emitters A and C were adjusted separately in both lateral position and angle. The alignment procedure is given in the text.

shown in Figure 8. With this in mind, the alignment procedure involved 3 simple steps, with the two emitters/ fibres /(shadow-) detectors being labelled $A$ and $C$, as shown in Figure 5: -

1. The emitter-Housing towards the rear of the suspension (A) was centered approximately on its fibre. It then was fixed in this position from below, using clamping screws.

2. The A-C detector housing was moved laterally, i.e., back-and-forth in the $\xi$-direction of Figure 1 (using a fine-pitch screw), in order to centre the shadow of fibre A onto its corresponding dual-photodiode detector within the Housing, and

3. emitter-Housing $\mathrm{C}$ was rotated about a vertical axis by up to $\pm 1.5^{\circ}$, so as to cast fibre C's shadow onto the centre of its respective dual-photodiode detector, before being clamped in position from below. No adjustment about the $\xi$-axis was required, since the plane in which the detectors lay was accurately vertical (like the fibres, themselves), whilst the housingsand so optical axes_ - of the emitters was set up to be close to horizontal.

This last step compensated for variations in the $30 \mathrm{~mm}$ nominal pitch of the two suspension fibres, by up to $\pm 0.5 \mathrm{~mm}$.

\section{Very low-noise current sources for the emitters}

\subsection{The current-source}

The primary goal of the complete VM shadow-sensor system was to have the maximum sensitivity to shadow displacement over a frequency range encompassing the expected fundamental VM mode of a highly loaded suspension (at approximately $500 \mathrm{~Hz}$ ), up to (say) its 
MST-101172.R2

$10^{\text {th }}$ harmonic, or $5 \mathrm{kHz}$. At the same time, it became clear that in order to meet, or exceed, the desired displacement sensitivity of $100 \mathrm{pm}(\mathrm{rms})$ per $\sqrt{\mathrm{Hz}}$, a noise performance as close as possible to the fundamental shot noise limit of its detectors was required. In this regard, the current source supplying the LEDs was found to play an important rôle.

Parenthetically, that the measured noise level might correspond closely to the fundamental shot noise limit was found not to be the case with an earlier example of NIR emitter, in which the current-source supplying the LEDs was provided by a MOSFET (NDT455N) transistor-based current source, in place of the bipolar transistor version described below. In that case, the noise Power Spectral Density (PSD) measured at the detector Amplifier's VM output was found to lie, on average, $+2.6 \mathrm{~dB}$ (a factor of $\times 1.35$ ) above the theoretical shot-noise limit. Clearly, this current-source for the emitter gave rise to correlated excess noise in bothimperfectly balanced-elements of the detector.

Indeed, in order to reduce the VM amplifier's output noise level, when connected to the shadow sensors, the column of $8 \times$ OP224 LEDs within each emitter was supplied, ultimately, by the ultra-stable, low-noise, constant current source shown in Figure 6 [14].

Seen from the non-inverting input of the LT1677 op-amp, in Figure 6, the voltage reference for the transistor was effectively a source voltage of $\mathrm{V}_{\text {source }}=1.00 \mathrm{~V}$, connected in series with a $1 \mathrm{k} \Omega$ resistor - this being the Thévenin equivalent of the LM4040AIZ-5.0 Voltage reference connected to the $3 \mathrm{k} \Omega$ and $750 \Omega$ potential-division resistors, and the two series-connected $200 \Omega$ resistors. Analysis of this circuit can be shown to yield the collector current for the transistor, $\mathrm{I}_{\mathrm{c}}$, in the following form: -

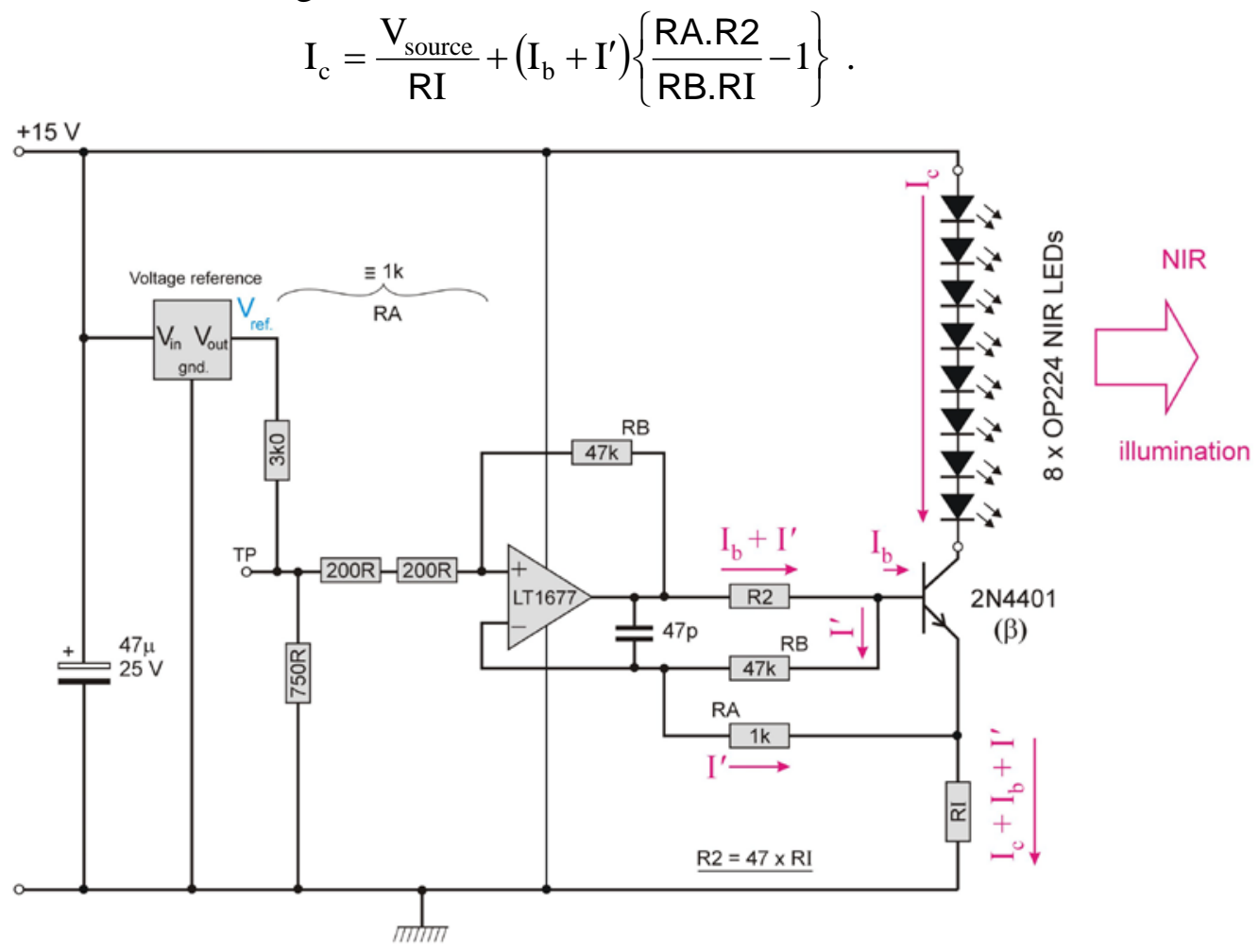

Figure 6. The low-noise, ultra-stable, $25 \mathrm{~mA}$ current-source in the Figure was used to supply the columns of $8 \times$ OP224 series-connected NIR LEDs (shown in Figure 4) in each emitter. By using this form of transistor control, the current passing through the LEDs, marked $I_{c}$ in the Figure, was made independent of both the currents $I_{b}$ and the I' (as explained in the text). The low-noise $15 \mathrm{~V}$ supply for this current-source was itself a 'finessed' source, which effectively removed voltage regulator noise-as shown in Figure 7, and its caption. As each LED had a forward-voltage drop of approximately $1.4 \mathrm{~V}$, the collector-emitter voltage of the transistor was approximately $2.75 \mathrm{~V}$-keeping its power dissipation below $70 \mathrm{~mW}$. The 'Voltage reference' IC (LM4040AIZ-5.0) had an output of $\mathrm{V}_{\text {ref. }}=5.00 \mathrm{~V}\left( \pm 0.1 \%, 30 \mathrm{ppm} /{ }^{\circ} \mathrm{C}\right)$, and the resistor $\mathrm{RI}=40 \Omega$. The $47 \mathrm{pF}$ capacitor ensured stability of the opamp, given its two feedback paths. Four such current-sources were built, one for each NIR emitter. 
MST-101172.R2

In equation 1 , the transistor's collector current $I_{c}$ involves neither its $D C$ current gain $(\beta \sim 220$, typically), nor its base-emitter voltage. Instead, it has been cast in terms of the current $\mathrm{I}_{\mathrm{b}}$, the base current of the $2 \mathrm{~N} 4401$ bipolar transistor $(\sim 1 \mathrm{nV} / \sqrt{\mathrm{Hz}}$, broadband [15]), and the current I', which are both defined in the Figure. In Figure 6, the value of the resistor labelled RB was chosen such that RB $=47 \times \mathrm{RA}$; and the terms within the braces of equation 1 then were made to vanish by choosing $\mathrm{R} 2=47 \times \mathrm{RI}(=1.88 \mathrm{k} \Omega$, with $\mathrm{RI}=40 \Omega)$. In this way the collector current $\mathrm{I}_{\mathrm{c}}$ was made quite independent of the small currents $\mathrm{I}_{\mathrm{b}}$ and $\mathrm{I}^{\prime}$ (which both tend to decrease with increasing temperature, such that $\mathrm{I}^{\prime} \sim \mathrm{I}_{\mathrm{b}} / 10$ ). Consequently, the transistor's collector current was made to be both thermally very stable, and low-noise, with a value of: -

$$
\mathrm{I}_{\mathrm{c}}=\frac{\mathrm{V}_{\text {source }}}{\mathrm{RI}} \text {, giving } \mathrm{I}_{\mathrm{c}}=25 \mathrm{~mA} \text {. }
$$

The rated maximum continuous current for OP224 LEDs is $100 \mathrm{~mA}$, and the LEDs were run deliberately at this relatively low current level of $25 \mathrm{~mA}$ in order to extend their operational lives. This current source contributed significantly to the overall high performance of the VM shadow-sensors, at, and above, $500 \mathrm{~Hz}$.

\subsection{Wideband noise reduction for the current-source: "noise finessing."}

Voltage regulators contribute significant noise to analog circuits across the audio range of frequencies, although the VM amplifier, being differential, rejected some of this noise. The situation was improved for the emitters in this work by using the circuit shown in Figure 7 [15]. Here, the significant level of voltage noise from the $+15 \mathrm{~V}$ voltage regulator was strongly reduced by this circuit, over a bandwidth of approximately (a few) $\mathrm{Hz}-20 \mathrm{kHz}$, the attenuation factor being approximately $15 \mathrm{~dB}$ at $\sim 10 \mathrm{kHz}$ (the broad peak in the voltage regulator's noise spectrum). The resulting $+15 \mathrm{~V}$ 'low-noise' output of this circuit was used to power simultaneously all four current sources of the type shown in Figure 6, one source per emitter.

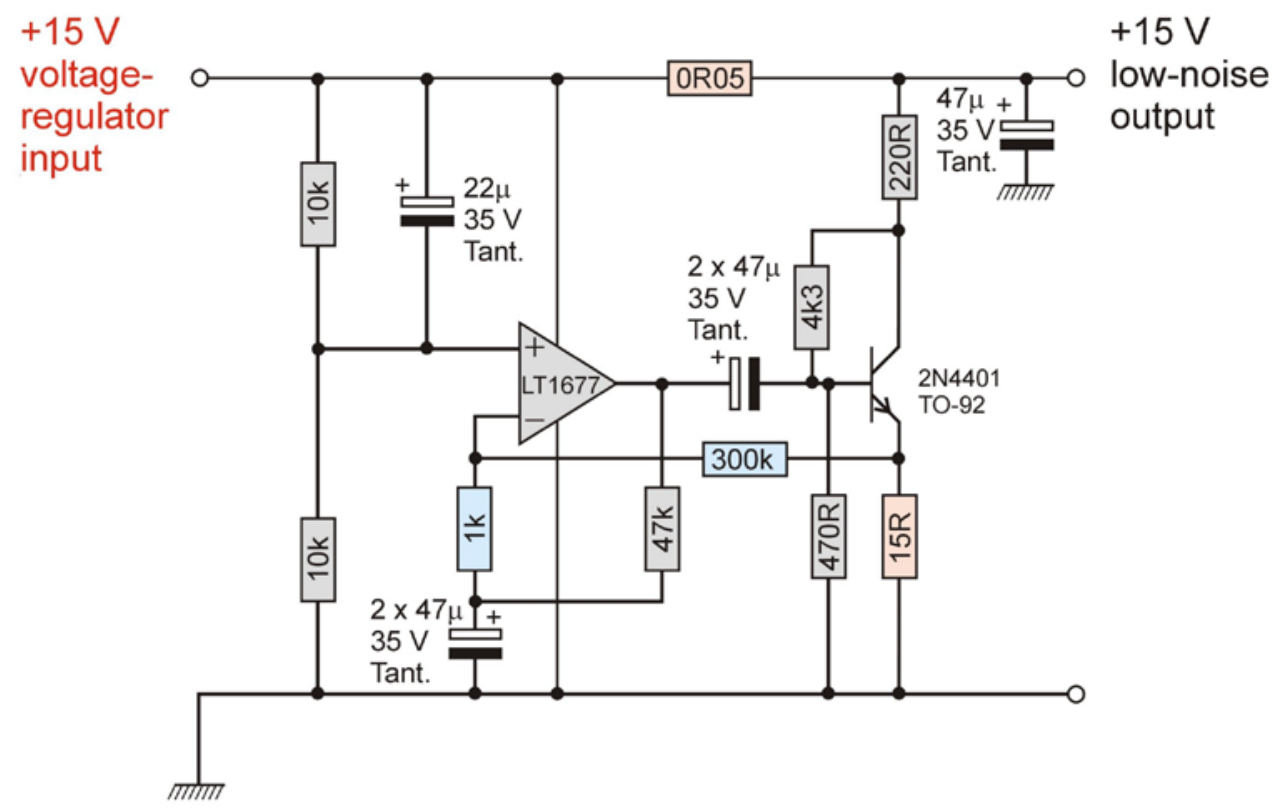

Figure 7. The voltage-regulator noise 'finessing' circuit [15]. In this circuit the transistor draws a nominal standing current of $24 \mathrm{~mA}$. The op-amp senses noise from the voltage regulator and, in response, forces the transistor to draw more or less current about its standing value, in order to cancel out the noise and create a very low-noise $+15 \mathrm{~V}$ output supply voltage, 'downstream' of the $50 \mathrm{~m} \Omega$ resistor. The paired 'bridge' resistors, $15 \Omega$ and $50 \mathrm{~m} \Omega$, and $300 \mathrm{k} \Omega$ and $1 \mathrm{k} \Omega$, stand in the ratio 300:1. 


\section{Violin-Mode fibre displacement calibration}

The displacement sensitivity and noise level of the $V M$ detection system was determined, of course, by the complete emitter/Fibre/detector/Amplifier assembly that was used to monitor VM resonances in each suspension fibre. The raw displacement responsivity was measured at the two $D C$ outputs of the Amplifier, whilst the displacement noise level was measured at the Amplifier's separate $A C$ output (please refer to Figure 8, which is reproduced here from reference [9], for clarity).

\subsection{The triple-output Amplifier}

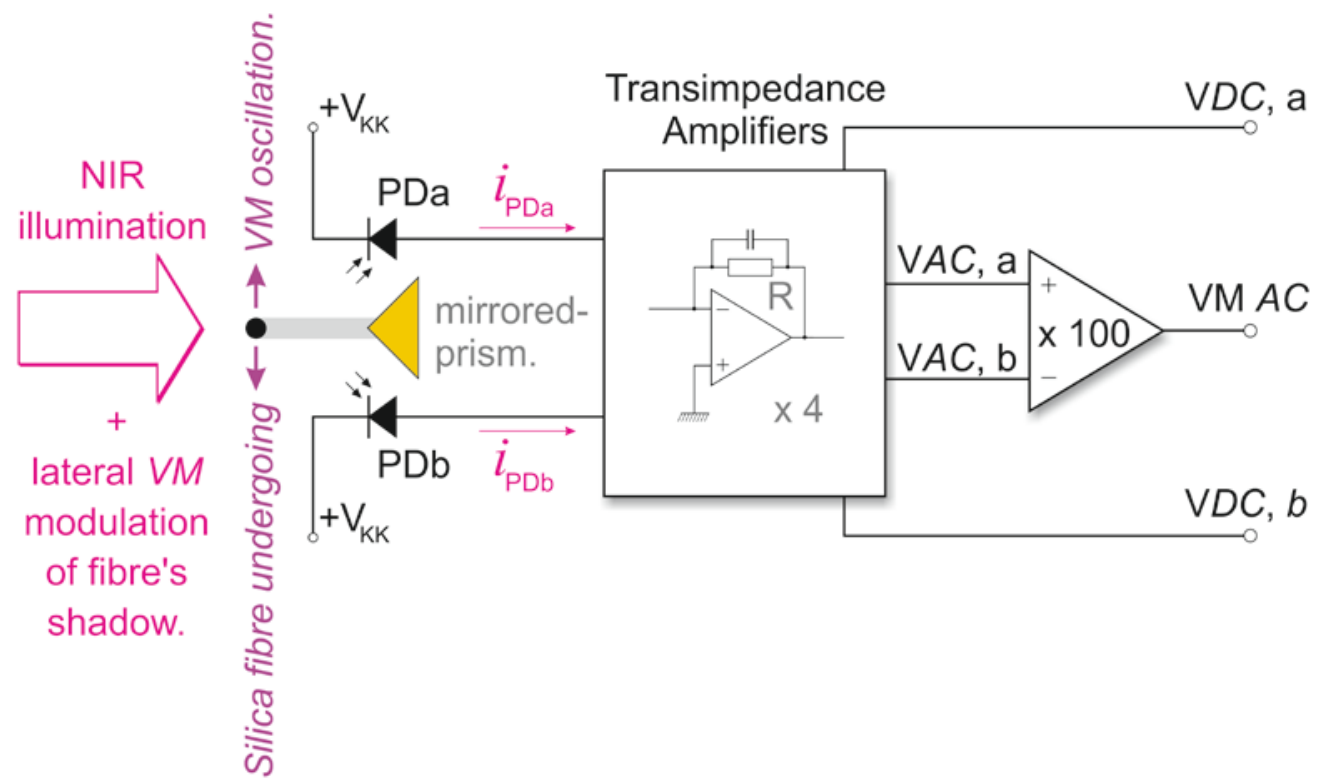

Figure 8. 'Plan view' schematic of the VM shadow-sensor, including its Transimpedance Amplifiers, for an individual silica suspension fibre. In the Figure, the emitter's collimated Near InfraRed beam illuminates the suspension fibre from the left, such that the fibre's shadow falls across the facing ridge of a $45^{\circ}-90^{\circ}-45^{\circ} \mathrm{Au} / \mathrm{Cr}$ mirror-coated beam-splitting prism. The reflected (split) NIR beam then falls onto photodiode elements PDa and PDb, generating in these elements $D C$ photocurrents $I_{\mathrm{a}}$ and $I_{\mathrm{b}}$, and anti-phase $A C$ photocurrents $i_{\mathrm{a}}$ and $i_{\mathrm{b}}$, respectively, where $i_{\mathrm{PDa}}=I_{\mathrm{a}}+i_{\mathrm{a}}$ and $i_{\mathrm{PDb}}=I_{\mathrm{b}}+i_{\mathrm{b}}$. The $D C$ and $A C$ (Violin-Mode) components of the photocurrents were amplified separately to yield the (positive) $D C$ output voltages $\mathrm{VDC}, \mathrm{a}$ and $\mathrm{VDC}, \mathrm{b}$, and the intermediate $A C$ voltages $\mathrm{VAC}$, a and $\mathrm{VAC}, \mathrm{b}$. The difference between these latter voltages was further amplified by a factor of (approximately) 100, to yield the single Violin-Mode $A C$ output, VM AC (please refer to the text).

All four detectors in the VM shadow-sensing system of this work comprised two (effectively) adjacent 'split-photodiode' elements, labelled, generically, PDa and PDb. The reverse bias for each element was $\mathrm{V}_{\mathrm{kk}} \sim 0.5 \mathrm{~V}$, and each of them was connected to its own detection (transimpedance-) amplifier system, having a separate $D C$ output, as indicated in blockdiagram form in Figure 8. In the Figure, the two $D C$ outputs are labelled $V D C$, $a$ and $V D C, b$, and the (positive) voltages at these outputs were direct analogues of the $D C$ photocurrents, $I_{\mathrm{a}}$ or $I_{\mathrm{b}}$, flowing in the corresponding element-via a transimpedance gain of $\mathrm{R}=120 \mathrm{k} \Omega$.

A silica fibre (and its shadow), undergoing transverse vibrations, generated $A C$ modulations of these photocurrents, and the $A C$ photocurrent components $i_{\mathrm{a}}$ and $i_{\mathrm{b}}\left(=-i_{\mathrm{a}}\right)$ flowed through separate transimpedance-amplifiers within the 'Transimpedance Amplifiers' block, generating in anti-phase the intermediate voltages $V A C, a$ and $V A C, b$, shown in the Figure. In this case, however, the transimpedance gain for each element was $\mathrm{R}=1.2 \mathrm{M} \Omega$. The difference between these latter voltages then was amplified with a differential gain of approximately $\times 100$, generating the third Amplifier output-the Violin-Mode AC output voltage signal, VM AC.

The frequency response of $V M A C$ was tailored so as to roll-off at a rate of $-40 \mathrm{~dB} / \mathrm{decade}$ below $226 \mathrm{~Hz}$, and above $8.93 \mathrm{kHz}$ (determining the $-3 \mathrm{~dB} \mathrm{VM}$ bandwidth), the mid-band frequency being at $1.48 \mathrm{kHz}$. Therefore, ostensibly, the mid-band AC (Violin-Mode) 
MST-101172.R2

transimpedance gain of the Amplifier was $\times 1000$ the $D C$ gain for that element, although the fairly narrow passband of the amplifier reduced this figure, very slightly; and its value at $500 \mathrm{~Hz}$ was somewhat lower still, because of the roll-off towards low frequencies-as discussed below.

\subsection{Optimizing the sensitivity to fibre-displacement}

In order to maximize the VM AC displacement sensitivity ('responsivity') of the shadow-sensor system, the $D C$ 'responsivity' for a particular emitter-detector configuration was maximizedthe (differential) $A C$ sensitivity to shadow displacement being approximately $1000 \times$ the (differential) DC sensitivity, mid-band. In fact, a calibration of the Amplifier using a NIR source of constant intensity, bearing a small ( $3 \%$ peak-peak, relative to the steady value) sinusoidal modulation at a known frequency, showed this factor actually to be $\times 976( \pm 4)$ midband, and $\times 904( \pm 4)$ at $500 \mathrm{~Hz}$.

In order first to find the $D C$ 'responsivity,' a vertically orientated $400 \mu \mathrm{m}$ diameter silica test fibre, approximately $150 \mathrm{~mm}$ long, was translated (in the $\xi$-direction of Figure 1) at a constant rate of a few tens of $\mu \mathrm{m}$ per second, using a $D C$-motor driven leadscrew-so as to pass transversely through the illuminating beam of a particular emitter. The position of the fibre was recorded continuously using a Renishaw LM10 magnetic position encoder, having a $1 \mu \mathrm{m}$ resolution, together with a microcontroller-based interface to eliminate jitter at low translationrates. The detector's two $D C$ voltages $V D C$, a and $V D C, \mathrm{~b}$, as shown in Figure 8, were recorded simultaneously at every $1 \mu \mathrm{m}$ step, with 12-bit resolution, using a National Instruments USM-6259 DAQ device, controlled by a LabView data acquisition program running on a Laptop PC. The detector's two DC output voltages were then plotted as a function of fibre position, producing a pair of 'shadow-notch' traces. The origin of the fibre's position $(\xi=0)$ was taken to be that point at which the shadow of the fibre fell equally onto $\mathrm{PDa}$ and PDb, where, in fact, the $D C$ (and so, also, $A C$ ) responsivity was found to be at a maximum. The two recorded voltages were differentiated off-line with respect to fibre position, $\xi$, and were then differenced, in order to generate the (differential) ' $D C$ responsivity' for that particular configuration of emitter and detector. This, typically, had values $\sim 10 \mathrm{kV} . \mathrm{m}^{-1}$, at the $\xi= \pm 0.1 \mathrm{~mm}$ points, i.e., at the ends of the useful $V M$ measurement range [9].

From the theory of shadow displacement detection [9] the ' $D C$ responsivity' can be written

$$
\frac{\partial(\mathrm{VDC}, \mathrm{a}-\mathrm{VDC}, b)}{\partial \xi} \propto \mathrm{I}_{0} \cdot \mathrm{g}_{\mathrm{CP}},
$$

where $I_{0}$ is the incident irradiance of the illuminating beam at the detector, and $g_{C P}$ is a dimensionless parameter describing the depth of the fibre's shadow along the dividing line between PDa and PDb $\left(g_{C P}=1\right.$ means no light reaches the detector along this line; $g_{C P}=0$ means an irradiance of $\mathrm{I}_{0}$ reaches the detector along this line).

In practice, moving the source of illumination (column of LEDs in the emitter) nearer to the fibre and detector increased $\mathrm{I}_{0}$ in equation 3 , but decreased $\mathrm{g}_{\mathrm{CP}}$, as the definition and contrast of the shadow gradually deteriorated. Masking the LEDs with a vertical slit narrower than their diameter certainly improved the shadow's contrast, but then the irradiance was severely reduced.

A number of both cylindrical and spherical collimating lenses, having different Focal Lengths in the range 100-63 mm, were used in order - effectively - to maximize the value of the product on the right-hand side of equation 3; and for each new optical arrangement the $D C$ responsivity was measured experimentally, as described above. The lens that was finally adopted is shown in Figure 2: an ' $80 \mathrm{~mm}$ FL (Focal Length)', spherical, plano-convex lens of BK7 glass was employed (FL $=81.5 \mathrm{~mm}$, at $\lambda=890 \mathrm{~nm}$ ), with the column of LEDs located at 
a distance of $57 \mathrm{~mm}$ from its first principal plane. The resulting beam was slightly divergent, since the apparent position of the emitter's light source was $214 \mathrm{~mm}$ from the fibre, rather than being at infinity (the optical path length from fibre to detector being $29.6 \mathrm{~mm}$ ). In this way, the $D C$ responsivity was increased by $\sim 20 \%$ over its value with the LEDs located at the focus of the lens. This was the configuration of the emitter that was used in the noise measurements, detailed below, and in the subsequent determination of VM displacement sensitivity for the full shadow-sensor system [9].

\section{Violin-Mode sensor noise level: comparison with theory}

The trace in Figure 9 shows the noise Power Spectral Density measured at the Amplifier's VM AC Output, under conditions of full illumination from Emitter D: here, no part of the illuminated fibre's shadow fell onto either of detector D's sensor elements (labelled PDa and $\mathrm{PDb}$, in Figure 8). Under these conditions the corresponding $D C$ photocurrents were found from the $V D C, a$ and $V D C, b$ outputs, indicated in Figure 8, to be $I_{\mathrm{a}}=61.82 \mu \mathrm{A}$, and $I_{\mathrm{b}}=57.13 \mu \mathrm{A}$. These photocurrents, along with their corresponding theoretically derived noise levels, are given in Table 1. As mentioned above, for each sensor the $A C$ transimpedance resistance (gain) was $\mathrm{R}=1.2 \mathrm{M} \Omega$, and the mid-band (differential) $A C$ gain of the following amplification stage was actually measured to be $\times 97.6$ (rather than $\times 100$ ), at $1.48 \mathrm{kHz}$. The detector's dark currents were negligible, in each case, being $~ 1 \mathrm{nA}$.

The anticipated frequency response of the VM AC Amplifier is indicated in Figure 9 by the dashed (blue) line, and, clearly, the measured noise PSD trace in the Figure followed closely the indicated theoretical transimpedance gain of this Amplifier down to $\sim 10 \mathrm{~Hz}$, below which $1 / \mathrm{f}$ noise dominated. The expected $-40 \mathrm{~dB}$ /decade Amplifier frequency response roll-offs towards low and high frequencies are also seen, reflected here in the noise PSD.

Theoretically, the mid-band differential $A C$ transimpedance gain of the VM AC Amplifier as a whole was $117.1 \mathrm{M} \Omega(=1.2 \mathrm{M} \Omega \times 97.6)$-i.e., only very slightly lower than the designed value of $120 \mathrm{M} \Omega$. If the full shadow-sensor system were limited in its sensitivity by $A C$ shot noise currents, where these arose from the standing $D C$ photocurrents flowing in the two sensor elements; and, further, if these noise currents were themselves uncorrelated, then the Amplifier's mid-band output noise level can be calculated as follows: -

The AC shot noise current in detector element PDa should be $i_{\mathrm{N}, \mathrm{a}}(\mathrm{rms})=\sqrt{2 \cdot q \cdot I_{\mathrm{a}}}$ [A $(\mathrm{rms}) /$ $\sqrt{ } \mathrm{Hz}]$, where $\boldsymbol{q}$ is the electronic charge, and $I_{\mathrm{a}}=61.82 \mu \mathrm{A}$. Similarly, for detector element PDb, $i_{\mathrm{N}, \mathrm{b}}(\mathrm{rms})=\sqrt{2 \cdot q \cdot I_{\mathrm{b}}}[\mathrm{A}(\mathrm{rms}) / \sqrt{\mathrm{Hz}}]$, where $I_{\mathrm{b}}=57.13 \mu \mathrm{A}$.

Therefore, if these two noise currents were uncorrelated, then their net effect can be summed (actually, via the differential amplifier of Figure 8) to yield an effective noise current, with a square-root-sum-of-squares value of: -

$i_{\mathrm{N}}(\mathrm{rms})=\sqrt{i_{\mathrm{N}, \mathrm{a}}^{2}(\mathrm{rms})+i_{\mathrm{N}, \mathrm{b}}^{2}(\mathrm{rms})},=6.174 \mathrm{pA}(\mathrm{rms}) / \sqrt{\mathrm{Hz}}$; and, given a common (in this analysis) transimpedance gain of 117.1 $\mathrm{M} \Omega$, such a noise current would equate to a final output noise from the Amplifier of $0.723 \mathrm{mV}(\mathrm{rms})$ per $\sqrt{\mathrm{Hz}}$, which can be expressed, alternatively, as $-62.82 \mathrm{dBV}$ (rms) $/ \sqrt{ } \mathrm{Hz}$.

This (uncorrelated) fundamental shot-noise limit is given in Table 1, and its level is indicated also in Figure 9-by the bold horizontal (red) line. Note that the actual measured mid-band noise PSD of the VM AC Amplifier lay at a level less than $0.1 \mathrm{~dB}$ above this fundamental shotnoise limit, for the un-shaded detector.

Nevertheless, with a silica fibre test sample placed between the emitter and detector, it was anticipated that - apart from any VM resonance-the relative noise level seen at the output of the detection system would increase, particularly with the fibre offset, laterally, at the end-of- 
range positions. The fibre, itself, would become a new conduit for noise. More importantly, the now differentially-shaded, unbalanced, detector would become far more susceptible to any common-mode 'intensity' noise from the emitter. Indeed, when this was tried the $\sim 150 \mathrm{~mm}$ long test fibre's proper (highly-air-damped) fundamental resonance was seen clearly, at a frequency of $\sim 300 \mathrm{~Hz}$, and the noise below $150 \mathrm{~Hz}$ was raised significantly in level—reaching $-38 \mathrm{dBV}(\mathrm{rms}) / \sqrt{ } \mathrm{Hz}$ at $43 \mathrm{~Hz}$; but this low-frequency noise, which exhibited many broad peaks, and was highly microphonic, was undoubtedly due to mechanical resonances in the test apparatus, and its steel enclosure, being transmitted to the fibre.

The single measurement point at $500 \mathrm{~Hz}$, shown by the cross in Figure 9, is representative of the noise above $\sim 300 \mathrm{~Hz}$, with the fibre at its offset end-of-range positions. Here, the noise PSD was found to have decreased relative to its un-shaded value-where no fibre was present in front of the detector. The end-of-range noise PSD was measured at a level of $(-64.2[6] \pm 0.2) \mathrm{dBV}(\mathrm{rms}) / \sqrt{\mathrm{Hz}}$, over 500 averages $-c f(-63.44 \pm 0.05) \mathrm{dBV}(\mathrm{rms}) / \sqrt{\mathrm{Hz}}$, for the same spot frequency, when the detector was un-shaded. Indeed, the average noise PSD at the Amplifier's output, under these end-of-range, differentially-shaded, conditions, and taken across all four shadow sensors of this work, was found to be a consistent $(-64.0 \pm 0.5) \mathrm{dBV}(\mathrm{rms}) / \sqrt{ } \mathrm{Hz}$, at this Violin-Mode frequency of $500 \mathrm{~Hz}$.

Typically, the reduction in average $D C$ photocurrent at these end-of-range (shaded) bias points was $17 \%$. If the measured noise level at $500 \mathrm{~Hz}$ were dominated still by shot noise in the detector, then a change in noise level of order $\sqrt{ } 0.83$ would be expected-equal to a factor of $-0.81 \mathrm{dBV}(\mathrm{rms}) / \sqrt{ } \mathrm{Hz}$. The measured change in noise level at the amplifier's $V M$ AC output was here equal to $(-0.82 \pm 0.25) \mathrm{dBV}(\mathrm{rms}) / \sqrt{\mathrm{Hz}}$, which was therefore quite consistent with the noise level from a detector, with its illuminated silica suspension fibre offset to one side, being essentially shot-noise limited still by the detector's two photodiode elements.

Had there been any significant common-mode illumination 'intensity' noise originating in the emitter, then it should have added to the detected noise level in the differentially-shaded case, but not in the un-shaded case. In this latter case, any common-mode illumination noise ought to have been rejected, largely, through the symmetry of the differential sensor-provided the levels of optical power incident on detector elements PDa and PDb were similar in value (and correlated in time). However, with the fibre offset from the centre of its detector, at the $\pm 0.1 \mathrm{~mm}$ positions, the detector's two photodiode elements were necessarily shaded by the fibre, but to different degrees. The $D C$ photocurrents flowing in PDa and PDb were proxies for the elements' respective levels of incident optical power, and in this offset case the photocurrents from, and illumination of, PDa and PDb were necessarily now quite different: please refer to Table 1. Indeed, when using the MOSFET-based current source mentioned in §5.1, the noise PSD was found to lie $+2.6 \mathrm{~dB}$ above the shot noise limit-as a result of illumination 'intensity' noise. However, when using the current source shown in Figure 6, any intensity noise contribution from the emitter was negligible, and so the detector was effectively shot noise limited in both the differentially-shaded and un-shaded cases.

\section{Conclusions}

The work presented here concerned the design and performance of Emitters for Violin-Mode (VM) shadow-sensors-which were designed to be retrofitted to the test-mass/mirror suspensions of aLIGO Gravitational Wave interferometers. It was intended that these sensors should monitor simultaneously any VM oscillations in the four $0.4 \mathrm{~mm}$ diameter by $600 \mathrm{~mm}$ long fused silica fibres of each suspension, over a bandwidth of $500 \mathrm{~Hz}-5 \mathrm{kHz}$. The required VM sensitivity of $100 \mathrm{pm}(\mathrm{rms}) / \sqrt{\mathrm{Hz}}$, at $500 \mathrm{~Hz}$, was challenging, and much effort was required to maximise the intrinsic sensitivity to $V M$ oscillations, whilst 'beating down' all potential sources of noise as far as was practicable—or, indeed, theoretically possible. 


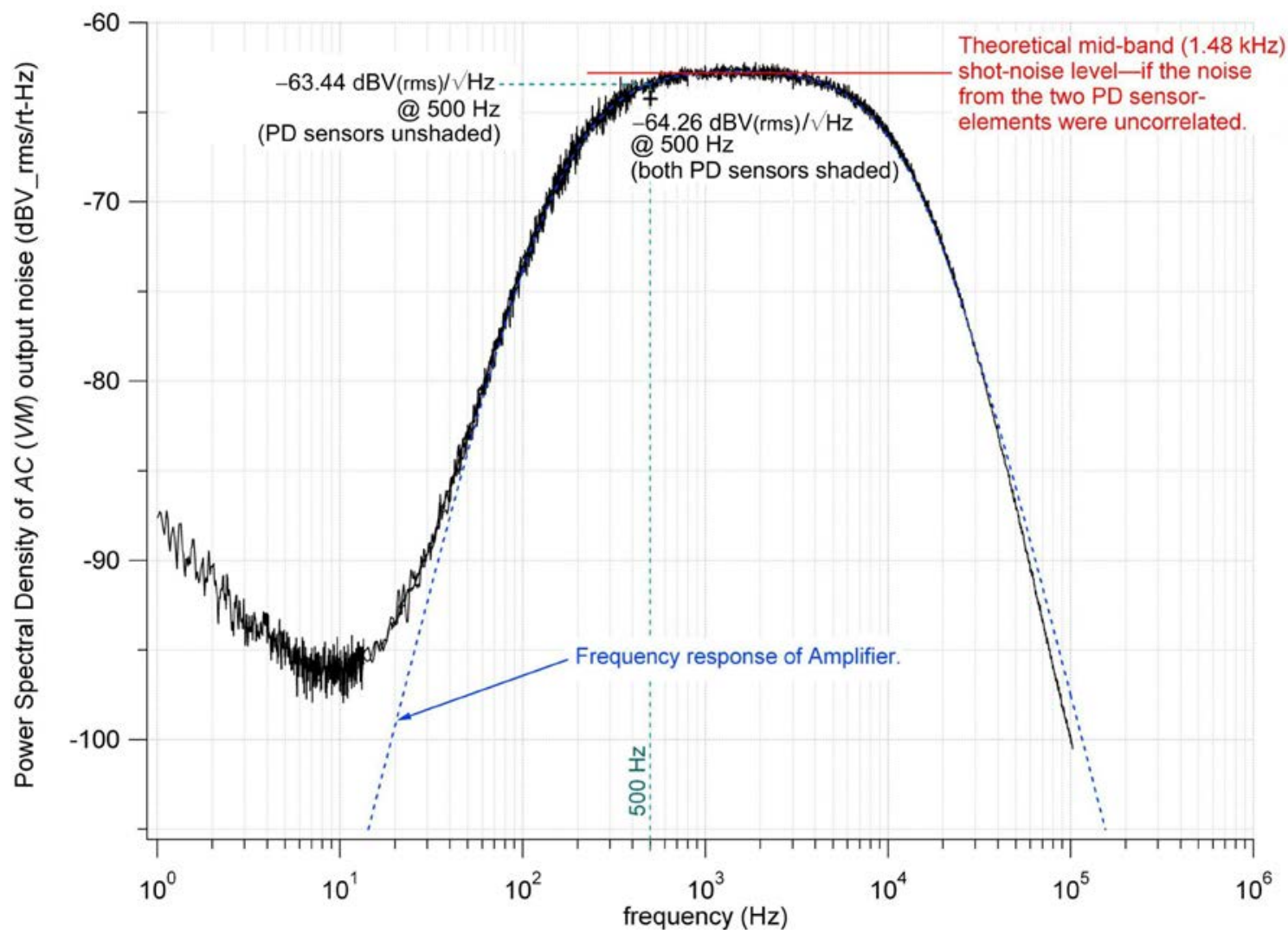

Figure 9. The trace in the Figure shows the wideband noise Power Spectral Density (PSD), measured at the Violin-Mode detector Amplifier's VM AC output (as shown in Figure 7), when the amplifier was connected to split-photodiode detector D. For comparison, the bold (red) horizontal line in the Figure, at -62.82 $\mathrm{dBV}(\mathrm{rms}) / \sqrt{ } \mathrm{Hz}$, indicates the fundamental mid-band noise level if the Emitter/detector/Amplifier system were shot-noise limited by the detector, alone. Here, Emitter and detector D were both mounted in a configuration similar to that shown in Figure 4, although the trace in this Figure shows the noise PSD under conditions where no part of the silica test fibre's shadow fell onto the detector. In contrast, when its shadow did fall onto the sensor elements - with the fibre at the $\pm 0.1 \mathrm{~mm}$ 'end-of-span' positions - the noise PSD at (e.g.) $500 \mathrm{~Hz}$ was found to be somewhat lower, as marked by the data point $(+)$ in the Figure (please refer to the text). The approximate fundamental Violin-Mode resonance frequency of an aLIGO suspension fibre is marked (500 $\mathrm{Hz})$, and the frequency response of the Amplifier is seen to accommodate up to at least the $10^{\text {th }}$ harmonic of this mode.

Table 1. The photocurrent values given in the Table correspond to different conditions of illumination of detector D by Emitter D: in the 'un-shaded' case the detector was wholly illuminated by the Emitter, whereas in the shaded case a silica test fibre was placed, at the $\xi=+0.1 \mathrm{~mm}$ (end-of-span) position, between the Emitter and detector, so that its shadow fell over both detector elements PDa, and PDb, although more over the latter-this being the rightmost of the two elements. In both cases the theoretical mid-band noise Power Spectral Density has been calculated at the output of the VM Amplifier, under the assumption that the noise level was due solely to uncorrelated $A C$ shot noise in the two photodiode elements of the detector.

\begin{tabular}{|c|c|c|c|}
\hline Detector & \multicolumn{2}{|c|}{ 'Split-photodiode' detector D's photocurrents } & $\begin{array}{c}\text { Theoretical shot noise } \\
\text { limited VM AC output }\end{array}$ \\
\hline$\underline{\text { Illumination Conditions }}$ & $\begin{array}{c}\text { DC photocurrent in } \\
\text { element PDa, } I_{\mathrm{a}} \\
(\mu \mathrm{A})\end{array}$ & $\begin{array}{c}\text { DC photocurrent in } \\
\text { element PDb, } I_{\mathrm{b}} \\
(\mu \mathrm{A})\end{array}$ & $\begin{array}{c}\text { Calculated mid-band } \\
(1.48 \mathrm{kHz}) \text { noise PSD } \\
(\mathrm{dBV}(\mathrm{rms}) / \sqrt{\mathrm{Hz}})\end{array}$ \\
\hline $\begin{array}{c}\text { Detector elements wholly } \\
\text { illuminated ('un-shaded'): }\end{array}$ & 61.82 & 57.13 & -62.82 \\
\hline $\begin{array}{c}\text { Fibre at the } \xi=+0.1 \mathrm{~mm} \\
\text { position, so both detector } \\
\text { elements were shaded } \\
\text { (PDb more than PDa): }\end{array}$ & 53.69 & 45.25 & -63.62 \\
\hline
\end{tabular}


To this end, the design of the emitter was first optimized, optically, in order to maximize the sensitivity to lateral displacement of silica test fibres (i.e., their shadows) in a complete VM shadow-sensing system. Each shadow-sensor was then tested for its final, output, noise level (Power Spectral Density), over the VM bandwidth. The noise tests themselves were carried out under two different conditions of illumination from the Emitter under test. In the first case, the silica test fibre was moved laterally, well out of the Emitter's illuminating beam, so that its shadow did not fall onto the shadow-displacement detector ('un-shaded' conditions); and in the second, the illuminated fibre was moved $\pm 0.1 \mathrm{~mm}$ away from a central position in front of its detector, so that the fibre's shadow fell onto both detector elements, but to intentionally different degrees ('shaded,' end-of-VM-detection-span, conditions). In both cases the Amplifier's VM AC output noise PSD was recorded (as in Figure 9). The results for detector D were presented here, since these were found to be typical of the four systems that were built and tested (and labelled A-D).

It is clear from the trace in Figure 9 that the mid-band $(1.48 \mathrm{kHz})$ un-shaded $A C$ noise level, measured at the Amplifier's VM AC output, lay very close indeed to the theoretically predicted level, were the measured noise to have arisen from uncorrelated shot noise in the two photodiode detector elements, alone. Indeed, if the noise from these two elements had been correlated, to some degree, then their combined level could have been up to $3 \mathrm{~dB}$ higher-a departure which would have been very obvious, given the ordinate scale in this Figure.

Noise arising from intensity fluctuations in the emitters, however, would have been strongly correlated, but the differential nature of the $A C$ Amplifier would not have rejected fully such noise, because the balance in the relative areas of the shadow-sensing photodiodes was only approximately $\pm(5-8) \%$ in each detector, even when un-shaded. Such noise was not seen at or above $500 \mathrm{~Hz}$, when using the current-source shown in Figure 6, although it is tempting to ascribe some of the 1 /f noise seen below $\sim 50 \mathrm{~Hz}$, in Figure 9, to this cause. It is certainly the case that the NIR LEDs used in the Emitters had a negative temperature coefficient of emitted flux, with a time-constant of tens of seconds.

Under shaded conditions, when the test fibre was at the $\xi= \pm 0.1 \mathrm{~mm}$ positions - with its shadow falling across the detector-the noise of the shadow-sensor at $500 \mathrm{~Hz}$ (say) was reduced, and any 'beam-wandering' or intensity-induced displacement noise from the Emitter must be considered negligible (for frequencies above $\sim 150 \mathrm{~Hz}$, at least).

Therefore, using the emitter described here, the Violin-Mode shadow-sensor system's overall noise performance was found to be very close to the fundamental shot-noise limited performance of the detectors themselves, under all practical conditions of illumination, and over the desired VM bandwidth-despite the rather low levels of DC photocurrent induced in the detector's sensor elements. These low levels of photocurrent were a direct result of the desirably (from the point of view of interference with the interferometer's operation) low irradiance at each detector from its corresponding Emitter $\left(2.8 \mathrm{~W} \cdot \mathrm{m}^{-2}\right)$. Using this design of Emitter source for illuminating the silica (suspension) test fibres, a noise PSD of $(-64.0 \pm 0.5) \mathrm{dBV}(\mathrm{rms}) / \sqrt{\mathrm{Hz}}$ was measured, taken across the four shadow-sensors, at $500 \mathrm{~Hz}$. The corresponding range of VM AC shadow- (fibre-) slope- (or transfer-) sensitivity was found to be: 8.27-10.59 MV (rms).m $\mathrm{m}^{-1}$ (rms), at $500 \mathrm{~Hz}$ [9].

Therefore, given the low noise level achieved for the VM system as a whole, via the use of this emitter and its current-source supply, the Violin-Mode detection sensitivity, taken over all four shadow-sensors, was encompassed by a displacement-sensitivity of (69 \pm 13 ) picometres (rms)/ $\sqrt{\mathrm{Hz}}$, at $500 \mathrm{~Hz}$, over a measuring span of $\pm 0.1 \mathrm{~mm}$ - exceeding the required target of $100 \mathrm{pm}$ (rms) $/ \sqrt{ } \mathrm{Hz}$, at this frequency. 
MST-101172.R2

At the time of writing the Violin-Mode sensor system described here has not been adopted for aLIGO, and, indeed, the need for VM damping has not yet been demonstrated. However, if it is found to be required, the current baseline solution is to use aLIGO's Arm Length Stabilisation system as a VM sensor [16]. In fact, the issue of vacuum compatibility remains unresolved for the VM sensor described here, because the Hamamatsu photodiodes used for the detector elements had been encapsulated, using an unknown epoxy. However, were it to become necessary, the issue of the epoxy for the photodiodes from this, or another, manufacturer probably could be resolved, and the LEDs and other components used are likely to prove vacuum compliant, or have vacuum-compliant alternatives.

\section{Acknowledgements}

We thank the IGR, University of Glasgow, Scotland, UK, for the silica fibre test samples used in this work, and acknowledge that without the research of Alan Cumming, Giles Hammond and Liam Cunningham of that institution on development and construction of the prototype aLIGO test-suspension used in obtaining the Violin-Mode results reported here, the work reported in this paper would not have been possible. We also thank Angus Bell of the IGR for assistance with the Violin-Mode measurements made on the IGR's aluminium test-mass suspension. Thanks go to Norna Robertson and Calum Torrie of Caltech and the IGR, Dennis Coyne of Caltech, Peter Fritschel, David Shoemaker, Rich Mittleman, and Brett Shapiro of MIT, Alberto Vecchio of the University of Birmingham, and Justin Greenhalgh of the CCLRC (RAL), for their oversight of, and assistance with, this work. We are grateful to the staff of the Physics Department's Electronics Workshop, and the Science Faculty's Mechanical Workshop, at the University of Strathclyde, for their careful construction of most of the component parts used in this work, and we would also like to thank the company Bar Knight Precision Engineers Ltd. of Clydebank, Scotland, for their very good, and timely, machining work. Finally, we are grateful for the support of grant STFC PP/F00110X/1, which sustained this work.

\section{References}

[1] Harry G M (for the LIGO Scientific Collaboration) 2010 Advanced LIGO: the next generation of gravitational wave detectors. Class. Quantum Grav. 27084006 (12pp).

[2] Raab F J et al 2004 Overview of LIGO Instrumentation Proceedings of SPIE 5500 1124 (29 Sept.).

[3] Abbott B P et al 2009 LIGO: The Laser Interferometer Gravitational-Wave Observatory Rep. Prog. Phys. 72076901.

[4] Aston S M et al 2012 Update on quadruple suspension design for Advanced LIGO Class. Quantum Grav. 29235004 (25pp).

[5] Heptonstall A et al 2011 Invited Article: $\mathrm{CO}_{2}$ laser production of fused silica fibers for use in interferometric gravitational wave detector mirror suspensions Rev. Sci. Instrum. 82011301 1-9.

[6] Cumming A V et al 2012 Design and development of the advanced LIGO monolithic fused silica suspension Class. Quantum Grav. 29035003 (18pp).

[7] Carbone, L., et al. 2012 Sensors and actuators for the Advanced LIGO mirror suspensions. Classical and Quantum Gravity 2911115005 (14pp).

[8] Dmitriev A et al 2010 Controlled damping of high-Q violin modes in fused silica suspension fibers. Class. Quantum Grav. 27025009 (8pp).

[9] Lockerbie N A and Tokmakov K V 2014 A 'Violin-Mode' shadow sensor for interferometric gravitational wave detectors (Joint, accompanying, paper: Measurement Science and Technology: 16pp). ibid (companion paper to this). 
[10] Lockerbie N A and Tokmakov K V 2009 Sensor for the Control and Damping of 'Violin-Mode’ Resonances in Silica suspension Fibres LIGO Document G0900588-v1.

[11] OP232 and OP224 LEDs: OPTEK Technology (a TT Electronics company), 1645 Wallace Drive, Carrollton, Texas 75006, USA.

[12] Lockerbie N A 2004 OP232 IR LED + SMD2420 Photodiode: output vs flag displacement LIGO Document No. T040178-00 available at https://dcc.ligo.org/.

(this document shows how the LED's noise changed in character, due to beamwandering, as the length of a light-guide was increased-making the light rays increasingly paraxial).

[13] Lockerbie N A et al 2011 First results from the 'Violin-Mode' tests on an advanced LIGO suspension, at MIT. Class. Quantum Grav. 28245001 (12pp).

[14] 2008 (March) Ged Drinkwater, of the Physics Department's Electronics Workshop, at the University of Strathclyde, Glasgow, Scotland, brought this current-source circuit to my attention (NAL).

[15] Finesse Voltage Regulator Noise! (Wenzel Associates Inc.). This document can be found at http://www.wenzel.com/documents/finesse.html.

[16] Instrument Science White Paper 2012 LIGO-T1200199-v2, p71 https://dcc.ligo.org. 